\title{
Increasing damping ratio of high speed aircraft by terminal sliding mode
}

\section{method}

\author{
Guangbin $\mathrm{Wu}^{1, \mathrm{a}}$ and Guoqiang Liang ${ }^{1}$ \\ ${ }^{1}$ Department of control engineering, Naval aeronautical and astronautical University \\ Yanti, 264001 \\ awuguangbin1024@126.com
}

Keywords: hypersonic aircraft; stability; control; free flying; numerical simulation

\begin{abstract}
A special method to increase damping ration of a kind of high speed vehicle was proposed by constructing a kind of terminal sliding mode surface. As the increase of damping ratio of flying system, the stability and stable margin can be greatly improved which is also a most important point cared by many aircraft designers. Although this kind of terminal method is not firstly proposed and is also not firstly applied in aircraft control, but it is mostly focused on the quickness point but not the damping ration. So this point is very new and although only simplified model is used to testify the rightness of proposed opinion, the detail simulation at last also can provide a strong foundation for the main conclusion.
\end{abstract}

\section{Introduction}

Since hypersonic concept is put forward, it have been vigorously studied in the world. The United States, Russia, The United Kingdom, Japan, France and other countries have made significant progress, the hypersonic flight technology will become an important direction of development in the field of Aeronautics and Astronautics in twenty-first Century, so it represents a country military force in some respects ${ }^{[1-4]}$.

Due to the complexity of the control technology, the high speed control has brought great challenges to the current control technology. And the biggest problem is that the robustness and stable margin of traditional control law is not big enough. So variable structure control has been widely used in the high speed control due to its strong robustness ${ }^{[5-8]}$. While terminal sliding mode is subject to the attention of scholars for its finite time convergence characteristics. Most scholars use its fast convergence characteristics, but in the case of reasonable parameters, terminal sliding mode can improve the system damping. Based on the above reason, a kind of terminal sliding mode control is applied in high speed aircraft control to improve the robustness of flying system.

\section{Model Description}

A kind of hypersonic vehicle can be simplified as below differential equations to described its pitch channel movement: 


$$
\begin{aligned}
& \dot{V}=\frac{T \cos \alpha-D}{m}-g \sin \gamma \\
& \dot{\gamma}=\frac{L+T \sin \alpha}{m V}-\frac{g \cos \gamma}{V} \\
& \dot{\alpha}=q-\dot{\gamma} \\
& \dot{q}=\frac{M}{I} \\
& \dot{h}=V \sin \gamma \\
& \ddot{\eta}=-2 \varsigma \omega_{n} \dot{\eta}-\omega_{n}^{2} \eta+\omega_{n}^{2} \eta_{c}, \omega_{n}=5, \varsigma=0.7
\end{aligned}
$$

Where aero coefficient is identified as

$$
\begin{gathered}
L=\bar{q} S C_{L}, C_{L}=C_{L}^{\alpha} \alpha+C_{L}^{\delta} \delta+C_{L}^{0}, \quad T=\bar{q} s C_{T} \\
D=\bar{q} S C_{D}, C_{D}=C_{d}^{\alpha^{2}} \alpha^{2}+C_{D}^{\alpha} \alpha+C_{D}^{\delta^{2}} \delta^{2}+C_{D}^{\delta} \delta+C_{D}^{0} \\
M=\bar{q} S \bar{c}\left[C_{M \alpha}+C_{M \delta}+C_{M q}\right], C_{M \alpha}=C_{M \alpha}^{\alpha^{2}} \alpha^{2}+C_{M \alpha}^{\alpha} \alpha+C_{M \alpha}^{0}, \\
C_{M \delta}=C_{e}\left(\delta_{e}-\alpha\right), \\
C_{M \alpha}=10^{-4}\left(0.06-e^{-M_{a} / 3}\right)\left(-2 \alpha^{2}+120 \alpha-1\right) \\
C_{M q}=\frac{\bar{c} q}{2 V}\left(-0.025 M_{a}+1.37\right)\left(-0.0021 \alpha^{2}+0.0053 \alpha-0.23\right) \\
C_{M \delta}=0.0292(\delta-\alpha) \\
C_{L}=\alpha\left(0.493+1.91 / M_{a}\right) \\
C_{D}=0.0082\left(171 \alpha^{2}+1.15 \alpha+2\right)\left(0.0012 M_{a}^{2}-0.054 M_{a}+1\right) \\
C_{T}=\left\{\begin{array}{l}
38\left[1-164\left(\alpha-\alpha_{0}\right)^{2}\right]\left(1+17 / M_{a}\right)(1+0.15) \eta, \eta<1 \\
38\left[1-164\left(\alpha-\alpha_{0}\right)^{2}\right]\left(1+17 / M_{a}\right)(1+0.15 \eta), \eta<1
\end{array}\right\}
\end{gathered}
$$

And $V$ is speed, $\gamma$ is the speed angle, $\alpha$ is attack angle, $Q$ is the attitude angle speed, $h$ is the height.

$\phi$ is the oil supplying factor, $\delta_{c}$ is the duck wing and $\delta_{e}$ is the lift rudder.

\section{Sliding mode controller design}

Define error variable as $e=\alpha-\alpha^{d}$, and choose a common integral sliding mode variable as

$$
S=\dot{e}+3 c_{1} e^{1 / 3}
$$


Solve the derivative of sliding mode as

$$
\dot{s}=\ddot{e}+c_{1} e^{-2 / 3} \dot{e}
$$

Then it can be transformed as

$$
\dot{s}=\ddot{\alpha}+c_{1} \dot{\alpha} e^{-2 / 3}
$$

Then

$$
\dot{s}=\dot{q}-\ddot{\gamma}+c_{1} e^{-2 / 3} q-c_{1} e^{-2 / 3} \dot{\gamma}
$$

By using the information of above model, it can be written as

$$
\dot{s}=\frac{M}{I}-\ddot{\gamma}+c_{1} q e^{-2 / 3}-c_{1}\left(\frac{L+T \sin \alpha}{m V}-\frac{g \cos \gamma}{V}\right) e^{-2 / 3}
$$

And it can be rewritten as

$$
\dot{s}=\frac{\left(\bar{q} S \bar{c}\left[C_{M \alpha}+C_{M \delta}+C_{M q}\right]\right)}{I}-\ddot{\gamma}+c_{1} q e^{-2 / 3}-c_{1} e^{-2 / 3}\left(\frac{L+T \sin \alpha}{m V}-\frac{g \cos \gamma}{V}\right)
$$

Finally, we get

$$
\begin{aligned}
& \dot{s}=\left(\left(\bar{q} S \bar{c}\left[10^{-4}\left(0.06-e^{-M_{a} / 3}\right)\left(-2 \alpha^{2}+120 \alpha-1\right)+0.0292(\delta-\alpha)\right) / I\right.\right. \\
& \left.\left.+\frac{\bar{c} q}{2 V I}\left(-0.025 M_{a}+1.37\right)\left(-0.0021 \alpha^{2}+0.0053 \alpha-0.23\right)\right]\right) \\
& -\ddot{\gamma}+c_{1} q e^{-2 / 3}-c_{1} e^{-2 / 3}\left(\frac{L+T \sin \alpha}{m V}-\frac{g \cos \gamma}{V}\right)
\end{aligned}
$$

And consider that the variable $L$ can also be written as functions of attack angle and actuator, then it can be written as follows:

$$
\begin{aligned}
& \dot{s}=\left(\left(\bar{q} S \bar{c}\left[10^{-4}\left(0.06-e^{-M_{a} / 3}\right)\left(-2 \alpha^{2}+120 \alpha-1\right)+0.0292(\delta-\alpha)\right) / I\right.\right. \\
& \left.\left.+\frac{\bar{c} q}{2 V I}\left(-0.025 M_{a}+1.37\right)\left(-0.0021 \alpha^{2}+0.0053 \alpha-0.23\right)\right]\right) \\
& -\ddot{\gamma}+c_{1} q e^{-2 / 3}-c_{1}\left(\frac{T \sin \alpha}{m V}-\frac{g \cos \gamma}{V}\right) e^{-2 / 3}-\frac{c_{1} \bar{q} S e^{-2 / 3}\left(C_{L}^{\alpha} \alpha+C_{L}^{\delta} \delta+C_{L}^{0}\right)}{m V}
\end{aligned}
$$

And define new variables as follows

$$
\begin{gathered}
l_{2}(\alpha, q, e)=\frac{\left(\bar{q} S \bar{c}\left[10^{-4}\left(0.06-e^{-M_{a} / 3}\right)\left(-2 \alpha^{2}+120 \alpha-1\right)-0.0292 \alpha\right)\right.}{I} \\
\left.\left.+\frac{\bar{c} q}{2 V I}\left(-0.025 M_{a}+1.37\right)\left(-0.0021 \alpha^{2}+0.0053 \alpha-0.23\right)\right]\right) \\
-\ddot{\gamma}+c_{1} q e^{-2 / 3}-c_{1} e^{-2 / 3}\left(\frac{T \sin \alpha}{m V}-\frac{g \cos \gamma}{V}\right)-\frac{c_{1} e^{-2 / 3} \bar{q} S\left(C_{L}^{\alpha} \alpha+C_{L}^{0}\right)}{m V} \\
l_{1}=\frac{\bar{q} S \bar{c} 0.0292}{I}-\frac{c_{1} \bar{q} S C_{L}^{\delta}}{m V} e^{-2 / 3}
\end{gathered}
$$

Then the derivative of sliding mode can be written as 


$$
\dot{s}=l_{1} \delta+l_{2}(\alpha, q, e)
$$

Then the sliding mode control law can be designed as

$$
\delta=-\operatorname{sign}\left(l_{1}\right)\left[k_{1} s+k_{2} \frac{s}{|s|+k_{3}}+k_{4} s^{1 / 3}+k_{5} \int s d t\right]
$$

Where $k_{i}>0$, and assume there exist a big enough positive constant such $k_{6}$ and $k_{7}$ that $\left|l_{2}(\alpha, q, e)\right|<k_{6}|s|+k_{7}$, Choose a Lyapunov function as

$$
V=\frac{1}{2} s^{2}+\frac{k_{5}\left|l_{1}\right|}{2}\left(\int s d t\right)^{2}
$$

Then solve its derivative, it satisfies

$$
\begin{aligned}
& \dot{V}=s \dot{s}+k_{5}\left|l_{1}\right|\left(s \int s d t\right) \\
& =l_{1} \delta s+s l_{2}(\alpha, q, e)+k_{5}\left|l_{1}\right|\left(s \int s d t\right)
\end{aligned}
$$

And it can be written as

$$
\dot{V}=-\left|l_{1}\right|\left[k_{1} s^{2}+k_{2} \frac{s^{2}}{|s|+k_{3}}+k_{4} s^{4 / 3}+k_{5} s \int s d t\right]+s l_{2}(\alpha, q, e)+k_{5}\left|l_{1}\right|\left(s \int s d t\right)
$$

With the help of inequality method, it can be rewritten as

$$
\begin{aligned}
& \dot{V} \leq-\left|l_{1}\right|\left[k_{1} s^{2}+k_{2} \frac{s^{2}}{|s|+k_{3}}+k_{4} s^{4 / 3}+k_{5} s \int s d t\right]+k_{6} s^{2}+k_{7}|s|+k_{5}\left|l_{1}\right|\left(s \int s d t\right) \\
& =-\left|l_{1}\right|\left[k_{1} s^{2}+k_{2} \frac{s^{2}}{|s|+k_{3}}+k_{4} s^{4 / 3}\right]+k_{6} s^{2}+k_{7}|s|
\end{aligned}
$$

So it is easy to choose big enough parameters $k_{1}, k_{2}$ and $k_{3}$ such that

$$
\dot{V} \leq 0
$$

Then according to Lyapunov theory, we can prove $s \rightarrow 0$.

Since $c_{1}>0$, then it has

$$
\dot{e}=-c_{1} e^{1 / 3}
$$

Then if $e>0, \dot{e}<0$, when $e<0, \dot{e}>0$.

Choose another Lyapunov function as

$$
V_{a}=e^{2} / 2
$$

Then it holds

$$
\dot{V}_{a} \leq 0
$$


Then according to Lyapunov theory, we have $e \rightarrow 0$, then finally we can prove that the system is stable.

\section{Numerical Simulation and result analysis}

Set model parameters as

$$
\begin{gathered}
I_{x x}=-7.1 * 10^{-5} \mathrm{~m}^{2}+19.1 \mathrm{~m}-59430 \\
I_{y y}=-8.03 * 10^{-4} \mathrm{~m}^{2}+219.74 m-1690000 \\
I_{z z}=-8.03 * 10^{-4} \mathrm{~m}^{2}+219.74 m-1690000 \\
I_{y y 0}=1.23 * 10^{7}, v_{s}=3.017 * 10^{2}, \rho_{a}=1.84 * 10^{2}, \\
g_{a}=9.7147, \quad h=30000, V=4525, \eta_{c}=0.15662
\end{gathered}
$$

With above model and air coefficients and set initial condition as above paragraph and write a program with $\mathrm{m}$ language in Matlab software, then the simulation can be done with above control law where desired value of attack angle is set as 4 degree, and simulation results can be shown as following figures.

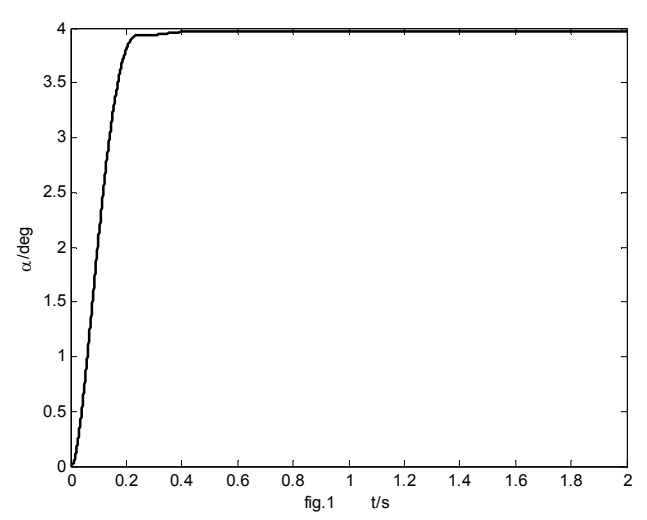

Fig 1 The curve of attack angle

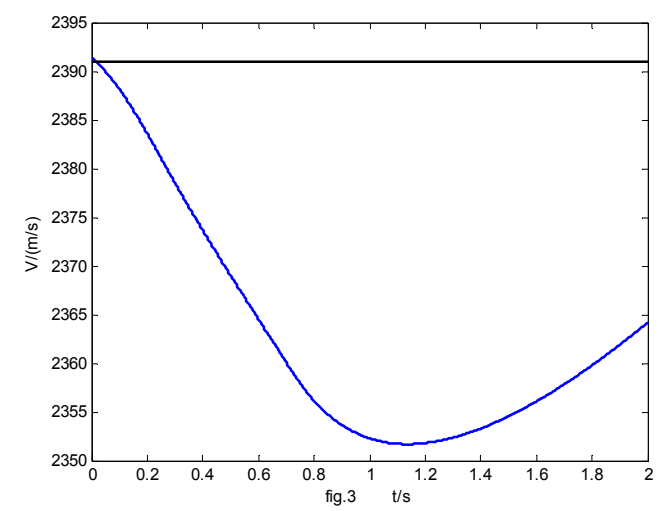

Fig 3 The curve of speed

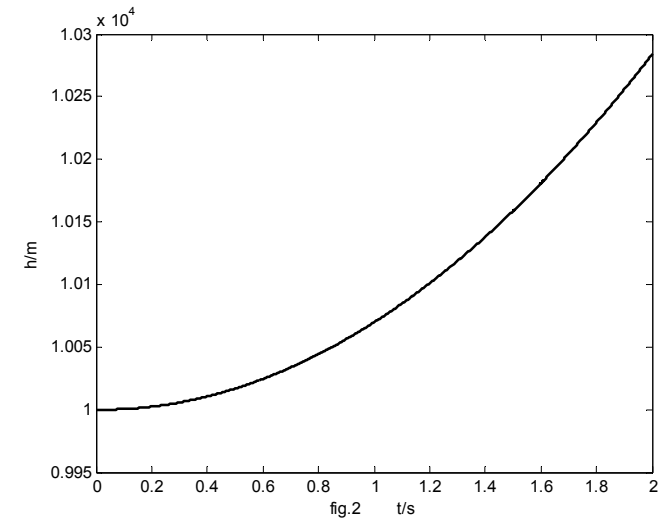

Fig 2 The curve of the height

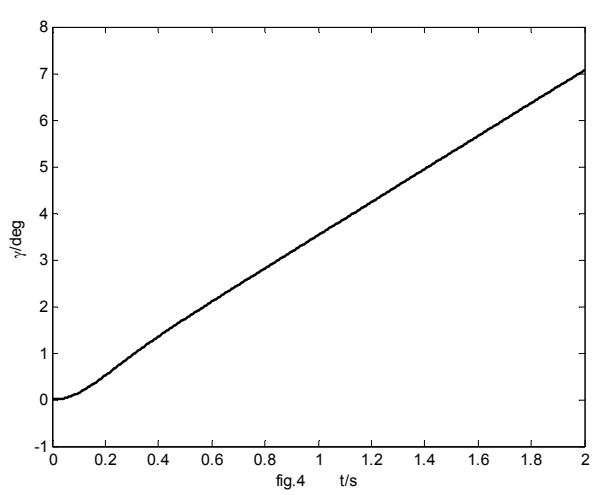

Fig 4 The curve of speed angle

From the above simulation results, it can be seen that the simulation results are obviously different from the traditional sliding mode because of the different structure of the terminal sliding mode compared with the traditional sliding mode. The biggest difference is that the angle of attack can be achieved without overshoot, which shows that the terminal sliding mode can effectively increase the system damping and improve the stability 
of the system.

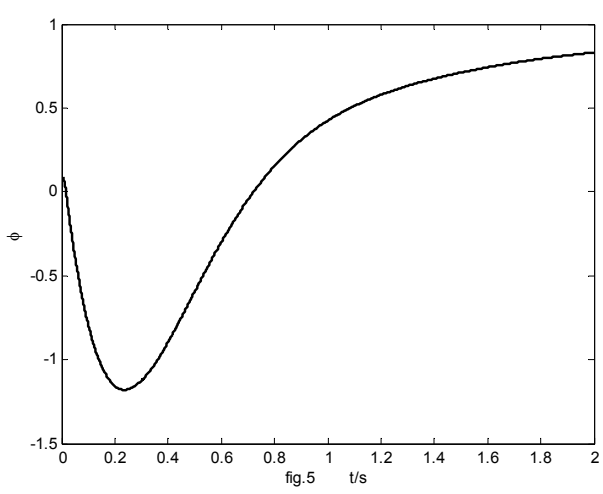

Fig 5 The curve of oil supplying factor

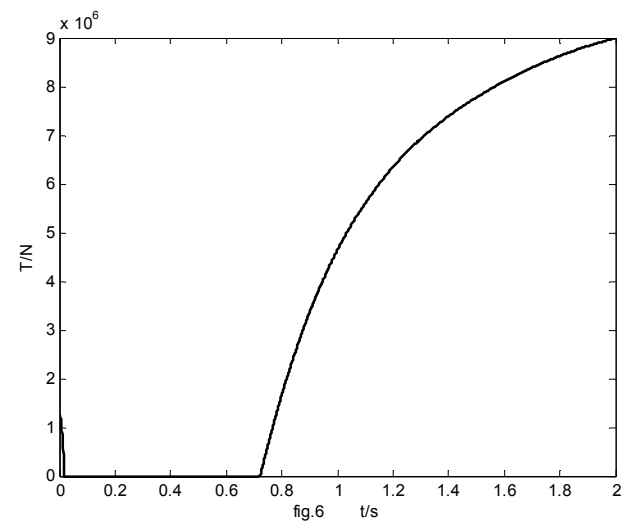

Fig 6 The curve of thrust

\section{Conclusion}

By introducing a kind of terminal sliding mode surface, a kind of terminal sliding mode controller was designed for the pitch channel model of high speed vehicle. Also the controller was proved to be stable by constructing two Lyapunov functions. And what is worthy pointing out is that the special constructing of terminal sliding mode can improve the system performance by increase system damping ratio which is also testified by the detailed simulation at last.

\section{References}

[1]Buschek $\mathrm{H}$ and Calise A J.Hypersonic flight control system design using fixed order robust controllers[A]. In:AIAA Guidance,Navigation and Control Conference[C].Chattanooga,TN, AIAA 95-6062

[2]Buschek H, Calise A J. Uncertainty modeling and fixed-order controller design for a hypersonicvehicle model. Journal of Guidance, Control and Dynamics, 1997, 20(1): 42-48.

[3]Heller M and Sachs G.Flight dynamics and robust control of a hypersonic test vehicle with ramjet propulsion[A].In:AIAA International Space Planes and Hypersonic Systems and Technologies Conference[C].Norfolk,VA,AIAA 98-1521

[4]M. Ohno, Y. Yamaguchi, T. Hata, M. Takahama, Robust flight control law design for an automatic landing flight experiment [J], Control Engineering Practice, 1999, 7(9):1143-1152

[5]Naidu S D,Banda S S and Buffington J L.Unified approach to $\mathrm{H}_{2}$ and $\mathrm{H}_{\infty}$ optimal control of a hypersonic vehicle[A].In:Pro of the American control conference[C].San Diego, California,1999, vol. 4:2737-2741.

[6]Christopher I. Marrison. synthesis of robust control systems for a hypersonic aircraft decision and contro1. IEEE, 1994.4:3324-3329

[7]E. mooij. 1inear quadratic regulator reentry control-performance assessment using a taguchi approach. AIAA, international space planes and hypersonic systems and technologies conference. 1998: 665-677.

[8]Kevin. P. Groves, Andrea. Serranti, Stephen. Yurkovich. Anti-Windup Control for an Air-breathing Hypersonic Vehicle Model. AIAA, 2006-6557: 1-14. 